\title{
Tanshinone IIA attenuates angiotensin II-induced apoptosis via Akt pathway in neonatal rat cardiomyocytes
}

\author{
Hong-jye HONG ${ }^{1}$, Ju-chi LIU ${ }^{2}$, Tzu-hurng $\mathrm{CHENG}^{3, \#, *}$, Paul $\mathrm{CHAN}^{2, \#, *}$ \\ ${ }^{1}$ School of Chinese Medicine, College of Chinese Medicine, China Medical University, Taichung, Taiwan, China; ${ }^{2}$ Department of Medi- \\ cine, Taipei Medical University-Wan Fang Hospital, Taipei, Taiwan, China; ${ }^{3}$ Department of Biological Science and Technology, College of \\ Life Sciences, China Medical University, Taichung, Taiwan, China
}

\begin{abstract}
Aim: To examine the effects of tanshinone IIA, the main effective component of Salvia miltiorrhiza (known as 'Danshen' in traditional Chinese medicine) on angiotensin II (Ang II)-mediated cardiomyocyte apoptosis.

Methods: Rat neonatal cardiomyocytes were primarily cultured with Ang II or Ang II plus tanshinone IIA. Myocyte apoptosis was evaluated by caspase-3 activity and DNA strand break level with TdT-mediated dUTP nick-end labeling (TUNEL) staining. Western blot analysis was employed to determine the related protein expression and flow cytometry assay was used to determine the TUNEL positive cells and the intracellular reactive oxygen species (ROS) production. SiRNA targeted to Akt was used.

Results: Ang II (0.1 $\mu \mathrm{mol} / \mathrm{L})$ remarkably increased caspase-3 activity, TUNEL positive cells, and cleaved caspase-3 and cytochrome $c$ expression, but reduced $\mathrm{Bcl}-\mathrm{X}_{\mathrm{L}}$ expression. These effects were effectively antagonized by pretreatment with tanshione IIA (1-3 $\mu$ mol/L). Tanshinone IIA had no effect on basal ROS level, while attenuated the ROS production by Ang II. Interestingly, tanshione IIA significantly increased the phosphorylated Akt level, which was countered by the PI3K antagonist wortmannin or LY294002. Knockdown of Akt with Akt siRNA significantly reduced Akt protein levels and tanshinone IIA protective effect.
\end{abstract}

Conclusion: Tanshinone IIA prevents Ang II-induced apoptosis, thereby suggesting that tanshinone IIA may be used for the prevention of the cardiac remodeling process.

Keywords: apoptosis; angiotensin II; tanshinone IIA; Akt; neonatal cardiomyocytes; caspase 3; reactive oxygen species

Acta Pharmacologica Sinica (2010) 31: 1569-1575; doi: 10.1038/aps.2010.176; published online 22 Nov 2010

\section{Introduction}

Apoptosis or programmed cell death is thought to play a crucial role in a variety of pathological situations ${ }^{[1]}$. The importance of apoptosis in heart failure has been recognized for over a decade ${ }^{[2]}$. The chronic release of reactive oxygen species (ROS) has been recently linked to the development of left ventricular hypertrophy and progression of heart failure ${ }^{[3]}$. Activation of the local and systemic renin-angiotensin system is closely related to increased morbidity in heart failure ${ }^{[4]}$. Pharmacological blockade of the renin-angiotensin system is beneficial in patients with heart failure ${ }^{[4]}$. Experiments using cultured cardiomyocytes have demonstrated that apoptosis

\footnotetext{
\# These authors codirected the project and contributed equally to the work.

* To whom correspondence should be addressed.

E-mail thcheng@mail.cmu.edu.tw (Tzu-hurng CHENG); chanpaul@wanfang.gov.tw (Paul CHAN)

Received 2010-05-27 Accepted 2010-09-12
}

can be stimulated in vitro by angiotensin II (Ang II) ${ }^{[5,6]}$. Ang II induces cardiomyocyte apoptosis, which contributes to heart failure possibly through enhanced ROS production ${ }^{[6]}$. Therefore, it is important to develop agents that inhibit cardiomyocyte apoptosis induced by Ang II and, as a result, improve cardiac dysfunction.

Tanshinone IIA extracted from Danshen, a popular medicinal herb used in traditional Chinese medicine, exhibits a variety of cardiovascular activities, including vasorelaxation, and cardioprotective effects $^{[7-10]}$. However, the pretreatment effects and mechanisms of tanshinone IIA on cardioprotection are not well understood. Akt is known to regulate many survival pathways in cardiac cells ${ }^{[11]}$ and has been reported to preserve cardiac function and prevent cardiac injury ${ }^{[12]}$. Therefore, the present study was set to evaluate the protective effect of tanshinone IIA on Ang II-induced cardiomyocyte apoptosis and to identify whether the underlying mechanisms are associated with the Akt-dependent pathway. 


\section{Materials and methods}

\section{Chemicals and reagents}

Dulbecco's modified Eagle's medium (DMEM), fetal calf serum, and tissue culture reagents were purchased from Invitrogen Corporation (Carlsbad, CA, USA). 5(6)-carboxy-2' 7'-dichlorofluorescein diacetate (DCFH-DA) was from Molecular Probes Inc (Eugene, OR, USA). All other chemicals of reagent grade were obtained from Sigma-Aldrich Chemical Co (St Louis, MO, USA). Antibodies were purchased from Lab Frontier Co Ltd, Seoul, Korea (anti-GAPDH), Cell Signaling Technology, Inc, Danvers, MA, USA (anti-caspase-3, anti-Ser473 phospho-Akt, anti-Akt), and Santa Cruz Biotechnology, Santa Cruz, CA, USA (anti-cytochrome c, anti-Bcl- $\mathrm{x}_{\mathrm{L}}$ ). Tanshinone IIA (purchased from Santa Cruz Biotechnology) was dissolved in dimethyl sulfoxide (DMSO), and the DMSO content in all groups was $0.1 \%$.

\section{Cell culture}

Primary cultures of neonatal rat cardiomyocytes were prepared as previously described ${ }^{[13]}$. The study was conducted in accordance with the Declaration of Helsinki and/or with the Guide for the Care and Use of Laboratory Animals as adopted and promulgated by the United States National Institutes of Health and was approved by the Institutional Animal Care and Use Committee of China Medical University (LAC-940069). The purity of the obtained myocyte cultures $(>95 \%)$ was determined by immunofluorescence microscopy. We counted all nuclei stained by 4',6-diamidino-2-phenyindole (DAPI) (Sigma-Aldrich) and all cells that stained positive for a-actinin (Sigma-Aldrich). The culture medium was replaced after $24 \mathrm{~h}$ with serum-free medium consisting of DMEM (10 $\mu \mathrm{g} / \mathrm{mL})$, insulin $(10 \mu \mathrm{g} / \mathrm{mL})$, and $\operatorname{BrdU}(0.1 \mathrm{mmol} / \mathrm{L})$ and exposed to the agents as indicated.

\section{Caspase- 3 activity assay}

For the caspase- 3 activity assay, the caspase-3 substrate rhodamine-110 (Z-DEVD-R110) was used as a prefluorescent substrate. The activity of caspase-3 was determined using a commercially available kit (Promega; Madison, WI, USA) according to the manufacturer's instructions. Briefly, after 48-h treatments with Ang II, tanshinone IIA, Ang II+tanshinone IIA, or vehicle, the caspase- 3 reagent was added and incubated for $10 \mathrm{~h}$. Levels of release of rhodamine- 110 were measured with a luminescence spectrometer LS55 (Perkin-Elmer, Waltham, MA, USA) at an excitation wavelength of $499 \mathrm{~nm}$ and an emission wavelength of $521 \mathrm{~nm}$.

\section{TUNEL assay}

Ang II-mediated apoptosis in cardiomyocytes was detected with enzymatic labeling of DNA strand breaks, which were identified with terminal deoxynucleotidyl transferase-mediated deoxyuridine triphosphate nick end-labeling (TUNEL) stain. TUNEL staining was performed with a Cell Death Detection kit (Roche, Mannheim, Germany) according to the manufacturer's directions. The apoptotic ratio was measured by flow cytometry according to the manufacturer's instructions.

\section{Western blot analysis}

Western blot analysis was performed as previously described $^{[14]}$. Membranes were blocked in $10 \mathrm{mmol} / \mathrm{L}$ Tris (pH 7.5), $100 \mathrm{mmol} / \mathrm{L} \mathrm{NaCl}$, and $0.1 \%$ Tween 20 containing $5 \%$ nonfat dry milk, followed by incubation with the primary antibody. Membranes were washed three times and incubated with the appropriate horseradish peroxidase-conjugated secondary antibody (1:5000 dilutions), and enhanced chemiluminescence (Amersham Biosciences Corp, NJ, USA), and bands were quantified with densitometry.

\section{Flow cytometric assay of $2^{\prime}, 7^{\prime}$-dichlorodihydrofluorescein oxidation}

The determination of intracellular ROS production was based on the oxidation of $2^{\prime}, 7^{\prime}$-dichlorodihydrofluorescein (DCFH) to fluorescent $2^{\prime}, 7^{\prime}$-dichlorofluorescein (DCF), as described previously ${ }^{[15]}$. DCFH was added to the cells at a final concentration of $10 \mu \mathrm{mol} / \mathrm{L}$ and incubated for $30 \mathrm{~min}$ at $37^{\circ} \mathrm{C}$. The cells were then washed once with PBS and maintained in 1-mL culture medium. Following drug treatment, the medium was aspirated and the cells were washed twice with PBS, and then dissociated with trypsin. Cellular fluorescence was determined by flow cytometry (FACS-SCAN, Becton-Dickinson, Franklin Lakes, NJ, USA). Cells were excited with an argon laser at $488 \mathrm{~nm}$, and measurements were taken at $510-540 \mathrm{~nm}$.

\section{Short interfering RNA (siRNA) transfection}

Akt siRNAs were purchased from Santa Cruz Biotechnology. Akt siRNAs and mock control oligonucleotides were transfected using the Lipofectamine (Invitrogen) reagent according to the manufacturer's instructions. The final concentration of the Akt siRNAs for transfection was $100 \mathrm{nmol} / \mathrm{L}$. Transfected cells were washed with PBS, and then incubated in new culture media for an additional $48 \mathrm{~h}$ for Ang II treatment and Western blot assays.

\section{Statistical analysis}

Results are expressed as mean \pm SEM. Statistical analysis was performed using Student's $t$ test or analysis of variance (ANOVA) with the Prism version 3.00 for Windows (GraphPad Software, San Diego, CA, USA). A value of $P<0.05$ was considered to be statistically significant.

\section{Results}

The effect of tanshinone IIA on Ang II-induced cardiomyocyte apoptosis

Using TUNEL staining with flow cytometry, we observed the effects of Ang II at a series of concentrations (0.001, 0.01, 0.1, and $1 \mu \mathrm{mol} / \mathrm{L}$ ). Ang II at $0.1-1 \mu \mathrm{mol} / \mathrm{L}$ appeared to have the strongest action (Figure 1A). We also observed the effects of Ang II $(0.1 \mu \mathrm{mol} / \mathrm{L})$ at different incubation times $(12,24,36$, and $48 \mathrm{~h}$ ) and found that Ang II exerted the most significant actions in inducing apoptotic cell death at an incubation time of $48 \mathrm{~h}$ (Figure 1B). Therefore, we present the data obtained from $0.1 \mu \mathrm{mol} / \mathrm{L}$ Ang II and $48 \mathrm{~h}$ of incubation in this study. 
Recent work has supported a central role for members of the caspase family, especially caspase-3, as effectors of apoptosis $^{[16]}$. To examine whether tanshinone IIA attenuates apoptosis induced by Ang II, we measured the caspase-3 activity in cells pretreated with tanshinone IIA. As shown in Figure 1C, the caspase-3 activity in Ang II-treated cells (0.1 and $1 \mu \mathrm{mol} / \mathrm{L} ; 48 \mathrm{~h}$ ) was significantly increased compared with vehicle-treated cells. Cardiomyocytes pretreated with tanshinone IIA $(1,3$, and $10 \mu \mathrm{mol} / \mathrm{L})$ for $30 \mathrm{~min}$, followed by $0.1 \mu \mathrm{mol} / \mathrm{L}$ Ang II for $48 \mathrm{~h}$, significantly inhibited the activation of caspase-3 by Ang II (Figure 1D).

The protective effect of tanshinone IIA against Ang IIinduced apoptosis in cardiomyocytes was further examined using TUNEL staining with flow cytometry (Figure 2A). Cardiomyocytes were pretreated with tanshinone IIA for $30 \mathrm{~min}$, followed by $0.1 \mu \mathrm{mol} / \mathrm{L}$ Ang II for $48 \mathrm{~h}$. Treatment with Ang II $(0.1 \mu \mathrm{mol} / \mathrm{L})$ for $48 \mathrm{~h}$ increased the percentage of apoptotic cells (Figure 2A). Tanshinone IIA treatment alone did not affect normal cell survival. In contrast, the pretreatment with tanshinone IIA (3, and $10 \mu \mathrm{mol} / \mathrm{L})$ markedly decreased the number of apoptotic cells induced by Ang II (Figure 2A). The influence of tanshinone IIA on apoptotic markers, such as cleaved caspase, cytochrome $\mathrm{c}$, and $\mathrm{Bcl}-\mathrm{x}_{\mathrm{L}}$, was further evaluated by Western blot analysis (Figure 2B). As shown in Figure $2 \mathrm{~B}$, the levels of cleaved caspase- 3 and cytosolic cytochrome $c$ were greatly elevated in the cells treated with $0.1 \mu \mathrm{mol} / \mathrm{L}$ Ang II for $12 \mathrm{~h}$. Pretreatment with tanshinone IIA at $3 \mu \mathrm{mol} / \mathrm{L}$ significantly reduced the amount of cleaved caspase- 3 and cytosolic cytochrome $c$, as compared with that in Ang II-treated alone cells. In contrast, the expression of $\mathrm{Bcl}-\mathrm{x}_{\mathrm{L}}$ was reduced by Ang II treatment, which was also recovered by tanshinone IIA pretreatment. These results indicate that the pretreatment with tanshinone IIA inhibited Ang II-induced cardiomyocyte apoptosis.

\section{The influence of tanshinone IIA on Ang Il-induced ROS generation in cardiomyocytes}

To evaluate the mechanism of the protective effect of tanshinone IIA on Ang II-induced apoptosis, the influence of tanshinone IIA on Ang II-induced ROS generation was monitored. We examined whether tanshinone IIA prevents Ang II-induced ROS formation. Tanshinone IIA-pretreated cells were then treated with $0.1 \mu \mathrm{mol} / \mathrm{L}$ Ang II for $1 \mathrm{~h}$. The Ang II-induced increases in intracellular ROS were revealed by measuring the fluorescent intensities of DCF. As shown in Figure $3 \mathrm{~A}$ and $3 \mathrm{~B}$, pretreatment with tanshinone IIA or the ROS scavenger N-acetylcysteine (NAC; $5 \mathrm{mmol} / \mathrm{L}$ ) significantly inhibited Ang II-induced ROS production. These results indicate that the pretreatment with tanshinone IIA inhibited Ang II-induced ROS production in cardiomyocytes.
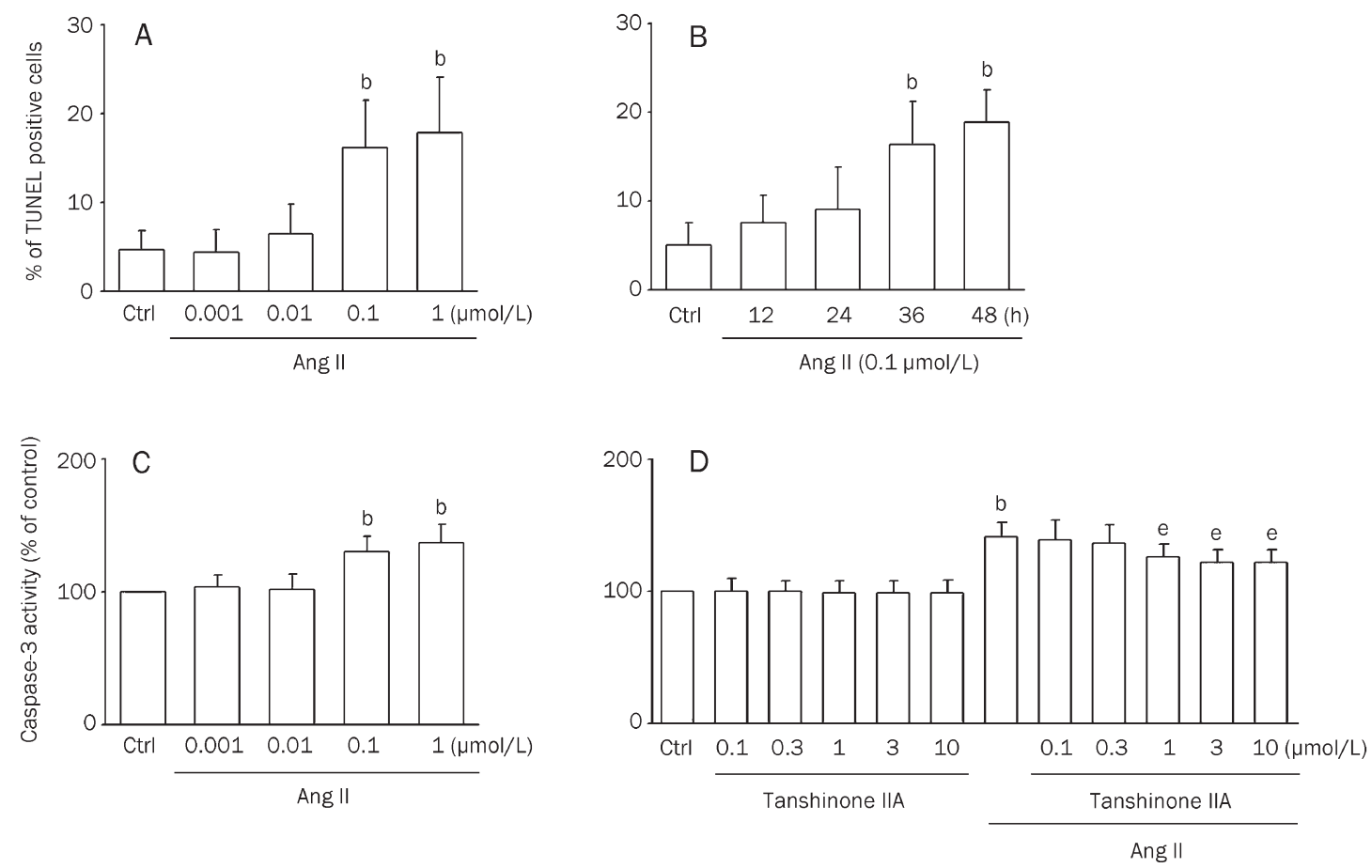

Figure 1. Caspase-3 activity in cardiomyocytes is inhibited by tanshinone IIA. Results were shown as mean \pm SEM. ${ }^{b} P<0.05$ vs control (Ctrl); ${ }^{e} P<0.05$ vs Ang II. (A) Bar graph showing the percentage of cardiomyocytes undergoing apoptosis in the presence of Ang II (0.001, 0.01, 0.1, and $1 \mu \mathrm{mol} / \mathrm{L})$ for $48 \mathrm{~h}$. TdT-mediated dUTP nick-end labeling (TUNEL) analysis was performed as described in Materials and methods. ( $n=5)(B)$ Bar graph showing the percentage of cardiomyocytes undergoing apoptosis in the presence of Ang II $(0.1 \mu \mathrm{mol} / \mathrm{L})$ at different incubation times $(12,24,36$, and $48 \mathrm{~h})$. ( $n=4)(\mathrm{C})$ Effects of angiotensin II (Ang II) $(0.001,0.01,0.1$, and $1 \mu \mathrm{mol} / \mathrm{L})$ on caspase-3 activity in cardiomyocytes. Caspase-3 activity was measured in lysates prepared from cardiomyocytes. $(n=4)(D)$ Cardiomyocytes pretreated with tanshinone IIA $(0.1,0.3,1,3$, and $10 \mu \mathrm{mol} / \mathrm{L}$; for $30 \mathrm{~min})$ in the absence or presence of $0.1 \mu \mathrm{mol} / \mathrm{L}$ Ang II for $48 \mathrm{~h}$. Bars indicate the intensity of R110 from 6 independent experiments, each in triplicate measurements. ( $n=8$ ) 


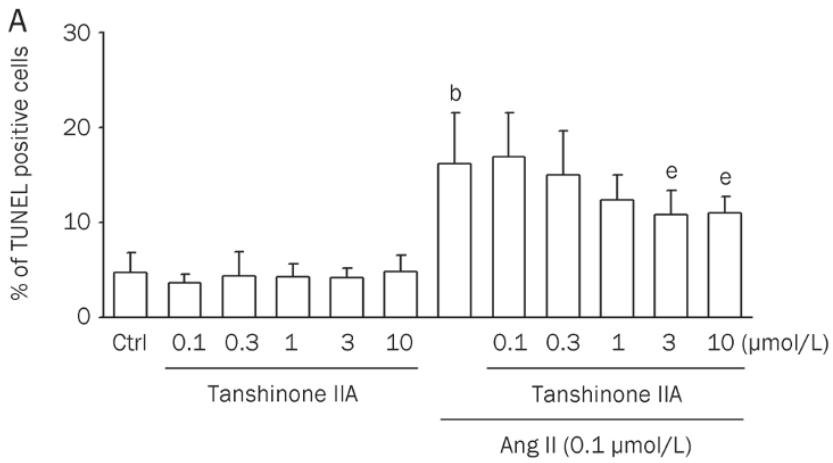

B
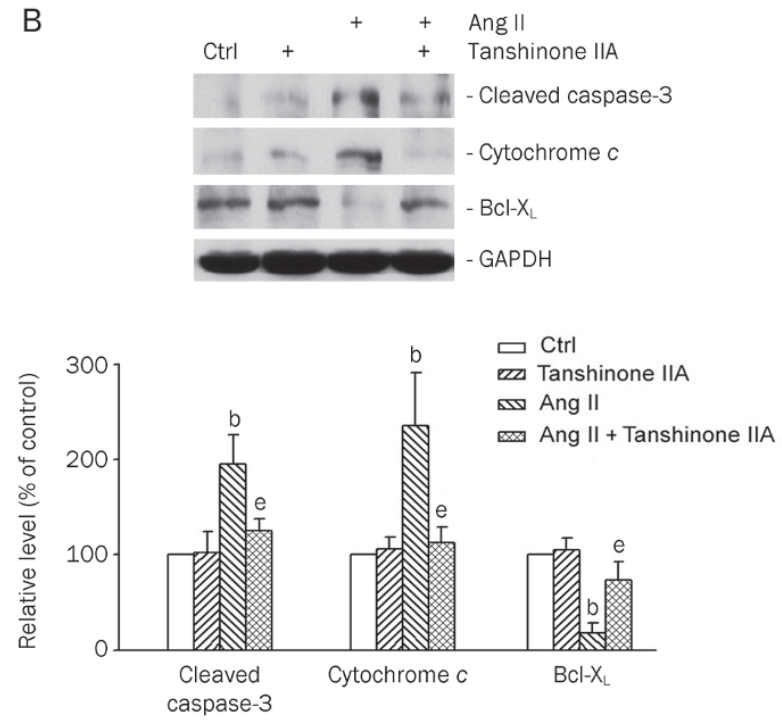

Figure 2. Tanshinone IIA protected cardiomyocytes from angiotensin II (Ang II)-induced apoptosis. (A) Flow cytometric analysis of TdT-mediated dUTP nick-end labeling (TUNEL)-stained cells. Cardiomyocytes pretreated with tanshinone IIA $(0.1,0.3,1,3$, and $10 \mu \mathrm{mol} / \mathrm{L}$; for $30 \mathrm{~min})$ in the absence or presence of $0.1 \mu \mathrm{mol} / \mathrm{L}$ Ang II for $48 \mathrm{~h}$. Percentages of apoptotic cardiomyocytes in the different groups. Results were shown as mean \pm SEM. ( $n=6)$. ${ }^{\mathrm{b}} P<0.05$ vs control (Ctrl); ${ }^{\mathrm{e}} P<0.05$ vs Ang II. (B) Effects of tanshinone IIA on apoptotic markers (cleaved caspase-3, released cytochrome $\mathrm{c}$, and $\mathrm{BcL}-\mathrm{x}_{\mathrm{L}}$ ) in Ang II-treated cardiomyocytes. The cells were pretreated with tanshinone IIA ( $3 \mu \mathrm{mol} / \mathrm{L})$ for $30 \mathrm{~min}$, and then treated with $0.1 \mu \mathrm{mol} / \mathrm{L}$ Ang II for $12 \mathrm{~h}$. Upper panels: Western blotting was carried out with the specific antibody against cleaved caspase-3, cytochrome $\mathrm{c}$, and $\mathrm{Bcl}-\mathrm{x}_{\mathrm{L}}$. Glyceraldehyde-3-phosphate dehydrogenase (GAPDH) was used as loading control. Lower panels: The quantitative results from the Western blots. Results were shown as mean \pm SEM. $(n=4)$. ${ }^{\mathrm{b}} \mathrm{P}<0.05$ vs control (Ctrl); ${ }^{\mathrm{e}} \mathrm{P}<0.05$ vs Ang II.

\section{Effects of tanshinone IIA on phospho-Akt in cardiomyocytes}

Akt is known to have an inhibitory effect on apoptosis in several cell types including cardiomyocytes ${ }^{[12]}$. To determine the effects of tanshinone IIA on Akt phosphorylation in rat cardiomyocytes, the amount of phospho-Akt (for serine 473) was measured. As shown in Figure 4A, tanshinone IIA $(3 \mu \mathrm{mol} / \mathrm{L})$ increased the serine phosphorylation of Akt from 5 to $60 \mathrm{~min}$ in cardiomyocytes. Since Akt is one of the
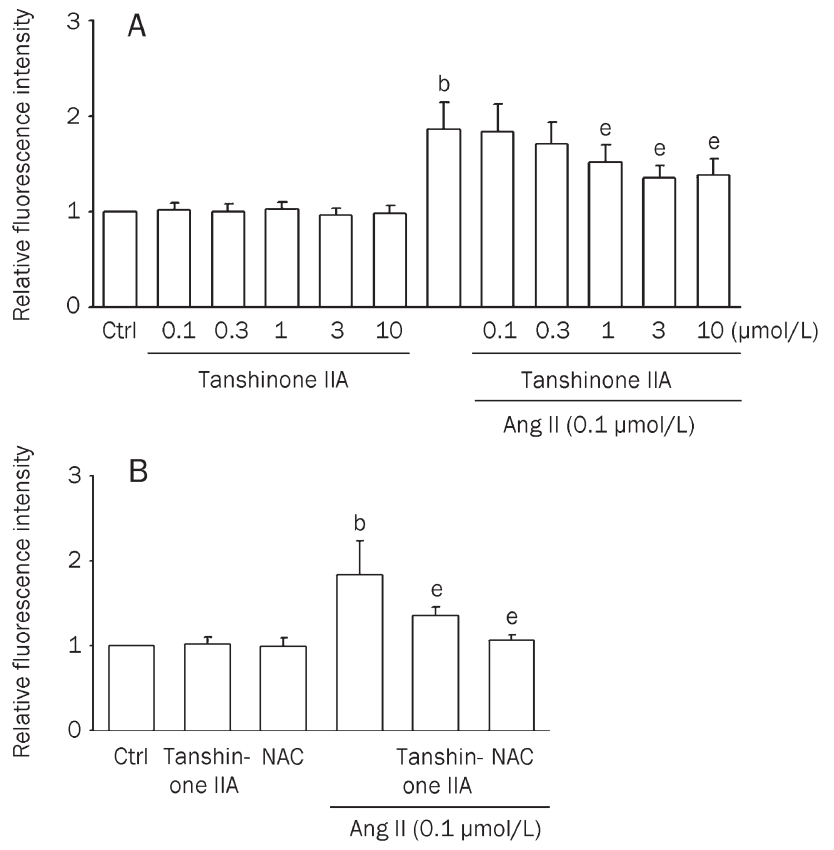

Figure 3. Effects of tanshinone IIA on angiotensin II (Ang II)-induced ROS generation in cardiomyocytes. Relative fluorescence intensity in rat cardiomyocytes was quantified by flow cytometry using dichlorofluorescin diacetate (DCFH-DA). The fluorescence intensities in untreated control cells are expressed as $100 \%$. Data were presented as relative intensity of the experimental groups compared to untreated control cells. Results were shown as mean \pm SEM. $(n=6)$. ${ }^{\mathrm{b}} P<0.05$ vs control (Ctrl); ${ }^{e} P<0.05$ vs Ang II. (A) Column bar graph of mean cell fluorescence for dichlorofluorescein (DCF) evaluated for cardiomyocytes pretreated with tanshinone IIA $(0.1,0.3,1,3$, and $10 \mu \mathrm{mol} / \mathrm{L}$; for $30 \mathrm{~min})$ and thereafter in the absence or presence of $0.1 \mu \mathrm{mol} / \mathrm{L}$ Ang II for $1 \mathrm{~h}$. (B) Cells were incubated with tanshinone IIA ( $3 \mu \mathrm{mol} / \mathrm{L})$ or $\mathrm{N}$-acetylcysteine (NAC) (5 $\mathrm{mmol} / \mathrm{L}$ ) and thereafter in the absence or presence of $0.1 \mu \mathrm{mol} / \mathrm{L}$ Ang II for $1 \mathrm{~h}$.

downstream effectors of PI3K, we next examined the effects of PI3K inhibitors on Akt phosphorylation. Pretreatment with the PI3K inhibitors wortmannin (Wort; $100 \mathrm{nmol} / \mathrm{L}$ ) and LY294002 (LY; $10 \mathrm{nmol} / \mathrm{L}$ ) inhibited the tanshinone IIAincreased Akt phosphorylation (Figure 4B). These findings indicate that tanshinone IIA induces Akt phosphorylation via the PI3K/Akt pathway. To confirm the involvement of Akt signaling pathway in tanshinone IIA survival actions, we next determined whether tanshinone IIA modified their degree of phosphorylation/activation in our experimental conditions. Figure 4C illustrates that incubation with tanshinone IIA enhanced Akt phosphorylation in cultures treated with Ang II. Compared with control cultures, Ang II alone did not modify Akt phosphorylation.

Role of Akt in the protective effect of tanshinone IIA on Ang IIinduced cardiomyocyte apoptosis

To identify the signaling pathways involved in the effect of tanshinone IIA, Akt siRNA, which mitigates the kinase activity of Akt, was transfected into cardiomyocytes. The Akt pro- 
A
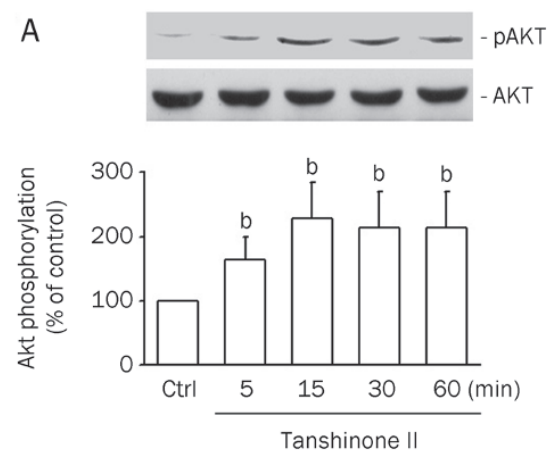

B
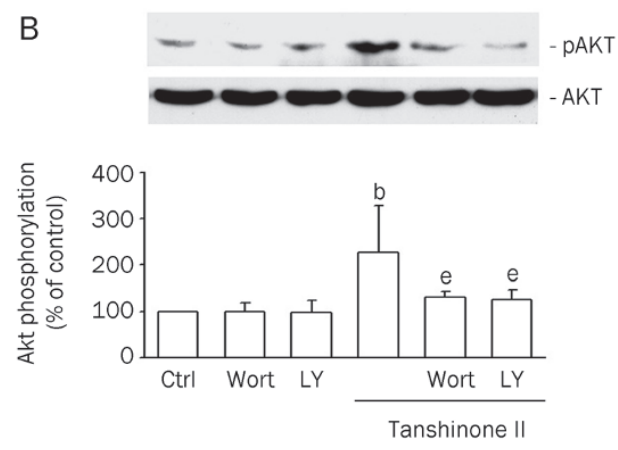

C
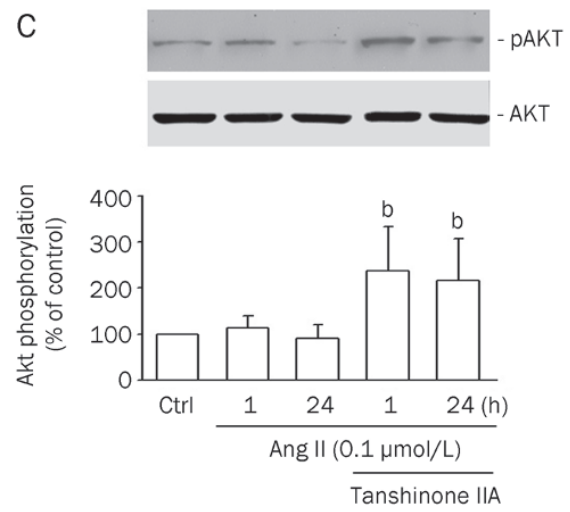

Figure 4. Tanshinone IIA induces Akt phosphorylation via PI3K. (A) Neonatal cardiomyocytes were incubated with $3 \mu \mathrm{mol} / \mathrm{L}$ tanshinone IIA for the indicated times. (B) Neonatal cardiomyocytes were incubated with wortmannin (Wort; $100 \mathrm{nmol} / \mathrm{L}$ ) or LY294002 (LY; $10 \mathrm{nmol} / \mathrm{L}$ ) for 30 min followed by incubation with $3 \mu \mathrm{mol} / \mathrm{L}$ tanshinone IIA for $15 \mathrm{~min}$. (C) Neonatal cardiomyocytes were incubated with tanshinone IIA $(3 \mu \mathrm{mol} / \mathrm{L})$ for 30 min prior to the addition of Ang II $(0.1 \mu \mathrm{mol} / \mathrm{L})$ for 1 or $24 \mathrm{~h}$. Western blot analyses were performed using site- and phospho-specific Akt antibodies against Ser473 (p-Akt, upper blot) or total Akt (lower blot). The results were shown as means \pm SEM $(n=4)$, expressed as percentage changes in phosphorylation over that in control cells. ${ }^{\mathrm{b}} P<0.05$ vs control (Ctrl). ${ }^{\mathrm{e}} \mathrm{P}<0.05$ vs tanshinone IIA.

tein levels were noticeably reduced by Akt siRNA transfection (Figure 5A). The inhibitory effect of tanshinone IIA on the Ang II-induced caspase-3 activation was partially reversed by Wort $(100 \mathrm{nmol} / \mathrm{L}), \mathrm{LY}(10 \mathrm{nmol} / \mathrm{L})$, and Akt siRNA (Figure 5B). Similarly, the inhibitory effect of tanshinone IIA on Ang
A

A
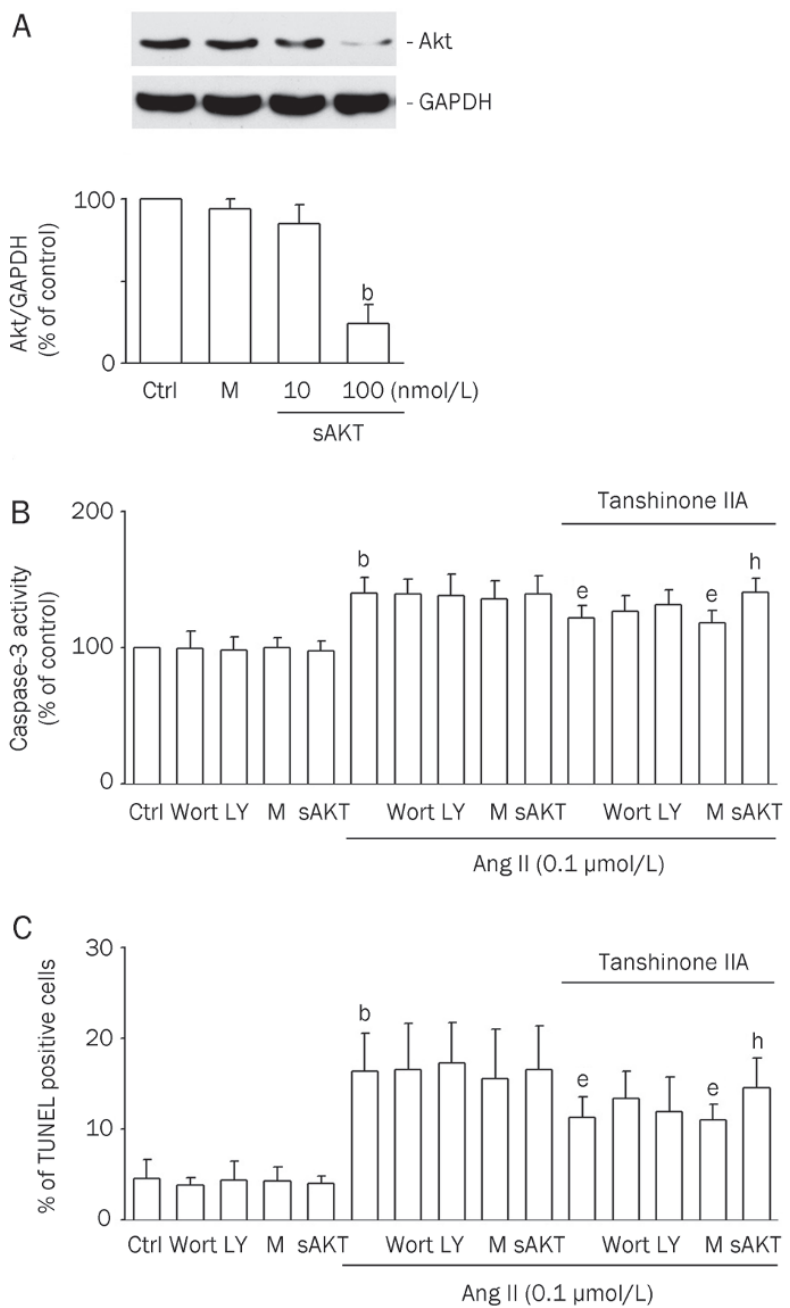

Figure 5. Blockage of the Akt pathway attenuated the inhibitory effect of tanshinone IIA on angiotensin II (Ang II)-induced apoptosis. Notes: Ctrl, untransfected control; M, mock control; sAkt, Akt siRNA transfection. ${ }^{\mathrm{b}} P<0.05$ vs the mock control. ${ }^{\mathrm{e}} P<0.05$ vs the Ang II treatment. ${ }^{\mathrm{h}} P<0.05$ vs the tanshinone IIA and Ang II treatment. (A) The effect of Akt siRNA transfection on Akt protein levels in cardiomyocytes. The cells were transfected with Akt siRNA (sAkt; 10, or $100 \mathrm{nmol} / \mathrm{L}$ ) to get Akt knockdown cells. Control siRNA was also applied as mock controls (M). Western blotting was carried out with the specific antibody against Akt. GAPDH was used as a loading control. Results were shown as mean \pm SEM $(n=3)$. (B) The effect of wortmannin (Wort), LY294002 (LY), and Akt siRNA on tanshinone IIA-decreased Ang II-induced caspase-3 activity in cardiomyocytes. Transfected cells were pretreated with or without tanshinone IIA ( $3 \mu \mathrm{mol} / \mathrm{L})$ for $30 \mathrm{~min}$, and then treated with Ang II (0.1 $\mu \mathrm{mol} / \mathrm{L}$ ) for $12 \mathrm{~h}$. Results were shown as mean $\pm \operatorname{SEM}(n=6)$. (C) The effect of Wort, LY, and Akt siRNA on tanshinone IIA-decreased Ang IIinduced apoptosis in cardiomyocytes. Transfected cells were pretreated with or without tanshinone IIA ( $3 \mu \mathrm{mol} / \mathrm{L})$ for $30 \mathrm{~min}$, and then treated with Ang II $(0.1 \mu \mathrm{mol} / \mathrm{L})$ for $48 \mathrm{~h}$. Results were shown as mean $\pm \operatorname{SEM}(n=6)$.

II-induced cardiomyocyte apoptosis was reduced by Wort (100 $\mathrm{nmol} / \mathrm{L}), \mathrm{LY}(10 \mathrm{nmol} / \mathrm{L})$, and Akt siRNA (Figure 5C). These results revealed the involvement of the Akt signaling pathway in tanshinone IIA's effect on Ang II-induced cardiomyocyte apoptosis. 


\section{Discussion}

The results of this study indicate for the first time that apoptosis in cardiomyocytes induced by Ang II can be considerably reduced (but not totally prevented) by tanshinone IIA. The mechanism involves the inhibition of apoptosis-related increase of ROS, activation of caspase-3, release of cytochrome $c$, and increased expression of Bcl- $\mathrm{x}_{\mathrm{L}}$. We also found that tanshinone IIA upregulated Akt phosphorylation, an interesting self-gain signaling that may possibly enhance the effect of tanshinone IIA. The causal relationship between upregulated Akt phosphorylation and tanshinone IIA action, however, needs further investigations.

The results of our study demonstrated that Ang II caused cardiomyocyte apoptosis; this finding is consistent with that of previous studies and shows that Ang II acts as an efficient inducer of apoptosis in adult and neonatal cardiomyocytes ${ }^{[5,6]}$. A statistically significant reduction of TUNEL-positive cardiomyocytes was observed when tanshinone IIA was added to Ang-II treated cells. Bcl- $\mathrm{x}_{\mathrm{L}}$ plays important roles in apoptotic cell death, whereas caspase-3 is a key downstream effector of apoptosis. To investigate the underlying mechanism(s) of the antiapoptotic effect of tanshinone IIA, we examined the expression of Bcl- $x_{\mathrm{L}}$ and caspase- 3 . The results showed that tanshinone IIA increased the expression of Bcl- $\mathrm{x}_{\mathrm{L}}$. We also found that the caspase- 3 activity of myocardial cells was significantly increased when cells were treated with Ang II and that tanshinone IIA greatly reduced this activation. The expression of $\mathrm{Bcl}-\mathrm{x}_{\mathrm{L}}$ and caspase- 3 was consistent with the results obtained by TUNEL staining with flow cytometry.

Tanshinone IIA is the main effective component of Salvia miltiorrhiza known as 'Danshen' in traditional Chinese medicine. Clinical evidence has shown that tanshinone IIA increases coronary blood flow and protects the heart against cardiac injury ${ }^{[17]}$. On the basis of the cardioprotective action of tanshinone IIA, we investigated the hypothesis that tanshinone IIA may prevent the death of cardiomyocytes. Cardiomyocyte apoptosis is one of the major pathogenic mechanisms underlying myocardial injury. Blocking the apoptotic process could prevent the loss of contractile cells and minimize cardiac injury induced by injury, thereby slowing down or even preventing the occurrence of heart failure ${ }^{[18]}$. Therefore, we performed TUNEL staining in order to explore the underlying mechanism responsible for the improvement of the cardiac function induced by tanshinone IIA. The results indicated that tanshinone IIA inhibited cardiomyocyte apoptosis induced by Ang II; this finding was similar to that of previous studies, which reported that tanshinone IIA protected cardiomyocytes against oxidative stress-triggered damage and apoptosis ${ }^{[8,18]}$. The possible mechanisms, which have been proposed for explaining the protective effects of tanshinone IIA, include antioxidant properties involving scavenging of free radicals in cardiomyocytes ${ }^{[7]}$. In addition, Akt, a serine/threonine kinase, is a primary mediator of the downstream effects of PI3K, which coordinates a variety of intracellular signals and regulates cell proliferation and survival. Recent studies have also shown that activation of the PI3K/Akt signaling pathway protects the myocardium from myocardial injury and prevents cardiomyocyte apoptosis ${ }^{[12]}$. In order to explore whether the protective effects of tanshinone IIA are associated with the Akt pathway, Akt siRNA was employed to compare the effects of co-administration of Akt siRNA and tanshinone IIA with the effects of administration of tanshinone IIA alone. Pretreatment with PI3K inhibitors (Wort and LY) or transfection with Akt siRNA abolished the cardioprotective effects of tanshinone IIA. These results suggest that tanshinone IIA induces cardioprotective effects through the activation of the Akt-pathway. The results of this study suggest that tanshinone IIA may offer a practicable approach to reduce apoptosis of cardiomyocytes and may merit further investigation. The present study strongly demonstrated that tanshinone IIA protects neonatal cardiomyocytes from Ang II-induced apoptosis. Tanshinone IIA might potentially be used to treat heart failure or other apoptosis-related heart diseases if further studies were performed to define and clarify the rationale for its clinical use.

\section{Acknowledgements}

This work was supported by a grant to Paul CHAN from the National Science Council, Taiwan, China (NSC 96-2320-B-038016-MY3) and a grant to Tzu-hurng CHENG from the China Medical University (CMU-98-S-22), Taichung, Taiwan, China.

\section{Author contribution}

Tzu-hurng CHENG and Paul CHAN designed the study and wrote the paper; Hong-jye HONG and Ju-chi LIU performed the study.

\section{References}

1 Masri C, Chandrashekhar Y. Apoptosis: a potentially reversible, metastable state of the heart. Heart Fail Rev 2008; 13: 175-9.

2 Satoh M, Matter CM, Ogita H, Takeshita K, Wang CY, Dorn GW 2nd, et al. Inhibition of apoptosis-regulated signaling kinase-1 and prevention of congestive heart failure by estrogen. Circulation 2007; 115: $3197-$ 204.

3 Sorescu D, Griendling KK. Reactive oxygen species, mitochondria, and $\mathrm{NAD}(\mathrm{P}) \mathrm{H}$ oxidases in the development and progression of heart failure. Congest Heart Fail 2002; 8: 132-40.

4 Paul M, Poyan Mehr A, Kreutz R. Physiology of local renin-angiotensin systems. Physiol Rev 2006; 86: 747-803.

5 Schroder D, Heger J, Piper HM, Euler G. Angiotensin II stimulates apoptosis via TGF-beta1 signaling in ventricular cardiomyocytes of rat. J Mol Med 2006; 84: 975-83.

6 Qin F, Patel R, Yan C, Liu W. NADPH oxidase is involved in angiotensin II-induced apoptosis in H9C2 cardiac muscle cells: effects of apocynin. Free Radic Biol Med 2006; 40: 236-46.

7 Zhou L, Zuo Z, Chow MS. Danshen: an overview of its chemistry, pharmacology, pharmacokinetics, and clinical use. J Clin Pharmacol 2005; 45: 1345-59.

8 Gao J, Yang G, Pi R, Li R, Wang P, Zhang H, et al. Tanshinone IIA protects neonatal rat cardiomyocytes from adriamycin-induced apoptosis. Transl Res 2008; 151: 79-87.

9 Sun DD, Wang HC, Wang XB, Luo Y, Jin ZX, Li ZC, et al. Tanshinone IIA: a new activator of human cardiac KCNQ1/KCNE1 $I_{\text {Ks }}$ potassium channels. Eur J Pharmacol 2008; 590: 317-21. 
$10 \mathrm{Xu} \mathrm{W,} \mathrm{Yang} \mathrm{J,} \mathrm{Wu} \mathrm{LM.} \mathrm{Cardioprotective} \mathrm{effects} \mathrm{of} \mathrm{tanshinone} \mathrm{IIA} \mathrm{on}$ myocardial ischemia injury in rats. Pharmazie 2009; 64: 332-6.

11 Shiraishi I, Melendez J, Ahn Y, Skavdahl M, Murphy E, Welch S, et al. Nuclear targeting of Akt enhances kinase activity and survival of cardiomyocytes. Circ Res 2004; 94: 884-91.

12 Matsui T, Tao J, del Monte F, Lee KH, Li L, Picard M, et al. Akt activation preserves cardiac function and prevents injury after transient cardiac ischemia in vivo. Circulation 2001; 104: 330-5.

13 Cheng TH, Shih NL, Chen SY, Wang DL, Chen JJ. Reactive oxygen species modulate endothelin-l-induced c-fos gene expression in cardiomyocytes. Cardiovasc Res 1999; 41: 654-62.

14 Chao HH, Liu JC, Hong HJ, Lin JW, Chen CH, Cheng TH. L-carnitine reduces doxorubicin-induced apoptosis through a prostacyclinmediated pathway in neonatal rat cardiomyocytes. Int J Cardiol 2009. doi: 10.1016/j.jijcard.2009.06.010.

15 Chen YL, Liu JC, Loh SH, Chen CH, Hong CY, Chen JJ, et al. Involvement of reactive oxygen species in urotensin Il-induced proliferation of cardiac fibroblasts. Eur J Pharmacol 2008; 593: 24-9.

16 Boatright KM, Salvesen GS. Mechanisms of caspase activation. Curr Opin Cell Biol 2003; 15: 725-31.

17 Jin UH, Suh SJ, Chang HW, Son JK, Lee SH, Son KH, et al. Tanshinone IIA from Salvia miltiorrhiza BUNGE inhibits human aortic smooth muscle cell migration and MMP-9 activity through AKT signaling pathway. J Cell Biochem 2008; 104: 15-26.

18 Fu J, Huang H, Liu J, Pi R, Chen J, Liu P. Tanshinone IIA protects cardiac myocytes against oxidative stress-triggered damage and apoptosis. Eur J Pharmacol 2007; 568: 213-21. 